\title{
CRISPRCasTyper: An automated tool for the identification, annotation and classification of CRISPR-Cas loci
}

\author{
Jakob Russel ${ }^{1 \#}$, Rafael Pinilla-Redondo ${ }^{12 \$}$, David Mayo-Muñoz ${ }^{3}$, Shiraz A. Shah ${ }^{4}$, Søren J. \\ Sørensen ${ }^{1 \#}$ \\ ${ }^{1}$ Section of Microbiology, University of Copenhagen, Copenhagen, Denmark. \\ ${ }^{2}$ University College Copenhagen, Copenhagen, Denmark. \\ ${ }^{3}$ Department of Microbiology and Immunology, University of Otago, Dunedin, New Zealand. \\ ${ }^{4}$ Copenhagen Prospective Studies on Asthma in Childhood (COPSAC), Herlev and Gentofte Hospital, University \\ of Copenhagen, Copenhagen, Denmark \\ $\$$ Contributed equally \\ \#Correspondence: \\ Jakob Russel: jakob.russel@bio.ku.dk \\ Sørenj.S Sørensen: sjs@bio.ku.dk
}

\section{Abstract}

CRISPR-Cas loci encode for highly diversified prokaryotic adaptive defense systems that have recently become popular for their applications in gene editing and beyond. The increasing demand for bioinformatic tools that systematically detect and classify CRISPR-Cas systems has been largely challenged by their complex dynamic nature and rapidly expanding classification. Here, we developed CRISPRCasTyper, a new automated software tool with improved capabilities for identifying and typing CRISPR arrays and cas loci across prokaryotic sequences, based on the latest classification and nomenclature (39 subtypes/variants) (Makarova et al. 2020; Pinilla-Redondo et al. 2019). As a novel feature, CRISPRCasTyper uses a machine learning approach to subtype CRISPR arrays based on the sequences of the direct repeats. This allows the typing of orphan and distant arrays which, for example, are commonly observed in fragmented metagenomic assemblies. Furthermore, the tool provides a graphical output, where CRISPRs and cas operon arrangements are visualized in the form of colored gene maps, thus aiding annotation of partial and novel systems through synteny. Moreover, CRISPRCasTyper can resolve hybrid CRISPR-Cas systems and detect loci spanning the ends of sequences with a circular topology, such as complete genomes and plasmids. CRISPRCasTyper was benchmarked against a manually curated set of 31 subtypes/variants with a median accuracy of $98.6 \%$. Altogether, we present an up-to-date and freely available software pipeline for significantly improved automated predictions of CRISPR-Cas loci across genomic sequences.

\section{Implementation}

CRISPRCasTyper is available through conda and $\mathrm{PyPi}$ under the MIT license (https://github.com/Russel88/CRISPRCasTyper), and is also available as a web server (http://cctyper.crispr.dk). 


\section{Introduction}

CRISPR-Cas systems constitute a group of bacterial and archaeal adaptive immune systems that have garnered much attention in recent years due to their promising biotechnological applications (Pickar-Oliver and Gersbach 2019; Barrangou and Doudna 2016). These systems are composed of two main components: 1) the CRISPR array, a chromosomal memory bank of sequences derived from previous infecting genetic parasites, and 2) operon(s) of CRISPR-associated (cas) genes encoding the proteins required during the three stages of immunity (adaptation, processing, and interference). For further details on the mechanisms driving the CRISPR-Cas immune response, we refer readers to recent reviews (Hille et al. 2018; Mohanraju et al. 2016; Jackson et al. 2017).

Like all other prokaryotic defense systems, CRISPR-Cas loci evolve rapidly in a constant arms race with their mobile genetic element foes (Hampton, Watson, and Fineran 2020). The resultant evolutionary tension has led to a remarkable diversification of CRISPR-Cas systems, which, together with the apparently frequent exchange of components and lack of a universal marker gene across systems (Koonin and Makarova 2019), greatly challenges the development of a unified classification scheme. Accordingly, classification efforts have relied on a multi-faceted approach that jointly takes into consideration the architectural organization of CRISPR-Cas loci, the presence/absence of certain Cas components, and sequence similarities of genes (Makarova et al. 2015). Broadly, the current classification contemplates two major classes, Class 1 and Class 2, that either rely on heteromeric multi-protein effector complexes or single multi-domain effector proteins, respectively (Makarova et al. 2020). In the next hierarchical level, there are six types (I, III and IV for Class 1; and II, V and VI for Class 2), each of which contain several subtypes and multiple variants. While recent years have seen an extraordinary expansion in the classification of newly discovered systems, the current classification is predicted to be nearly complete at the "type" level (Makarova, Wolf, and Koonin 2018; Makarova et al. 2020). For a summary of the state of the art classification and nomenclature, we refer the readers to recent comprehensive reviews and articles (Makarova et al. 2020; Pinilla-Redondo et al. 2019).

The systematic efforts to classify novel CRISPR-Cas systems have run parallel to those aiming their automated prediction across genomic sequences. Although systematic CRISPR-Cas identification pipelines have been developed (Crawley, Henriksen, and Barrangou 2018; Couvin et al. 2018; Lange et al. 2013), their sensitivity below the type level is generally inadequate. Furthermore, the discovery of novel systems has been occurring at a breakneck pace, rendering older classification software obsolete. Additionally, many CRISPR-Cas loci are complex, comprising hybrid cas cassettes or share arrays between different Cas types. For instance, recent work has shown that around $40 \%$ of CRISPR-Cas loci show atypical organizations, where orphan CRISPR arrays and cas operons are common, as well as hybrid loci resulting from the associations of different co-occurring systems within genomes (e.g. distinct types of interference modules and one shared adaptation cassette) (Bernheim et al. 2020). However, the CRISPR-Cas prediction tools published so far largely lack the formalism required to handle such complexity. 
Here we present CRISPRCasTyper, a new tool that can accurately identify and annotate CRISPR and cas loci automatically based on the newest classification (Makarova et al. 2020; Pinilla-Redondo et al. 2019). Besides classifying cas operons, CRISPRCasTyper can also accurately assign subtypes to CRISPR arrays based on the sequence composition of the consensus repeat. We also provide the first benchmark of automated classification of CRISPR-Cas loci on a manually curated dataset across all known subtypes, which exemplifies the strengths of CRISPRCasTyper.

\section{Software description}

CRISPRCasTyper identifies cas operons and associated CRISPR arrays from an input fasta-formatted DNA sequence (Fig. 1A). CRISPRCasTyper searches for cas and other genes functionally linked to CRISPR-Cas systems with HMMER3 (Eddy 2009) against 680 Hidden Markov Models (HMMs). Matches to Class 2 effectors (cas9, cas12, cas13) and the III-E gRAMP fusion protein are filtered by specific E-value and coverage cutoffs optimized specifically for each effector. The remaining HMM matches are filtered by overall cutoffs (see Methods and Materials for details). Adjacent cas and accessory genes are then joined into operons; inclusion of a gene in the operon is based solely on synteny. These operons are then typed based on a scoring scheme (see Methods and Materials for details).

CRISPRCasTyper includes the following 39 subtypes/variants: I-A, I-B, I-C, I-D, I-E, I-F, I-G, II-A, II-B, II-C, III-A, III-B, III-C, III-D, III-E, III-F, V-A, V-B, V-C, V-D, V-E, V-F, V-G, V-H, V-I, V-J, VI-A, VI-B, VI-C, VI-D (Makarova et al. 2020), IV-A1, IV-A2, IV-A3, IV-B, IV-C, IV-D, IV-E (Pinilla-Redondo et al. 2019), and transposon-associated V-K (Strecker et al. 2019) and I-F (Klompe et al. 2019).

To aid in resolving ambiguous CRISPR-Cas operons, and to subtype distant and orphan CRISPR arrays, we created a CRISPR repeat classification model. We used gradient boosting decision trees fitted to counts of canonical tetramers (Chen and Guestrin 2016, see Methods and Materials for details). Only subtypes with at least 20 known repeats were included. The classifier has a median accuracy across the 19 included subtypes of $89 \%$ on an unseen test dataset (Fig. 1B). Furthermore, the web server includes an additional model, which is automatically re-trained monthly using subtyped repeats crowdsourced from the inputs from web server users. This novel feature ensures that the accuracy of the tool increases over time and with usage of the platform, as well as its ability to recognize previously undetectable subtypes/variants. As of writing, this model includes more than $\sim 34 \mathrm{k}$ repeats from non-redundant genomes, in addition to the $\sim 6 \mathrm{k}$ repeats in the manually curated set. This model also includes the subtypes IV-D, IV-E, V-B, V-F, V-J, VI-A, and VI-D (Fig. S1), and has a median per-subtype accuracy of $84 \%$, mainly drawn down by the rare subtypes III-C, IV-D, and V-B.

As an additional feature, CRISPRCasTyper automatically draws gene maps to enable visualization of the operonic structure (Fig. 1C). These gene maps can be expanded to include HMM matches below the inclusion thresholds, which could aid the discovery of diversified cas components or accessory genes, partial and novel CRISPR-Cas variants/subtypes, especially around orphan CRISPR arrays. 
Moreover, CRISPRCasTyper can resolve loci spanning the ends of circular sequences, such that loci are not erroneously split in two due to the linear representation of circular sequences. Furthermore, the percentage completion of both the interference and adaptation modules of each detected operon are provided in the output. Finally, CRISPRCasTyper runs in less than a minute on a typical genome (2-6 Mbp) using 4 threads, and in less than 10 minutes on a deep metagenome assembly (60-100 Mbp) using 20 threads.
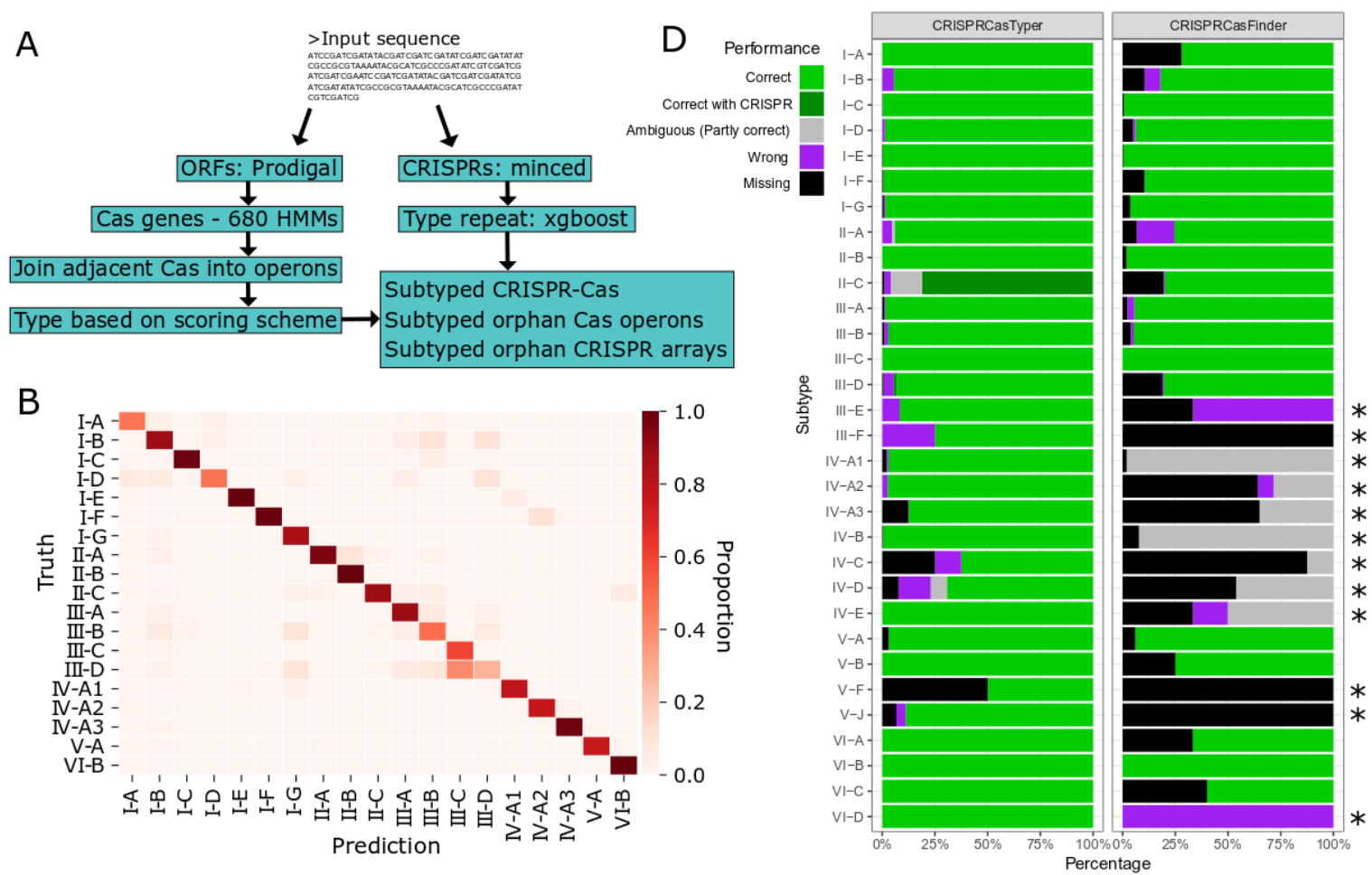

C

II-C: NZ_LS483446.1@1(24706-29792)
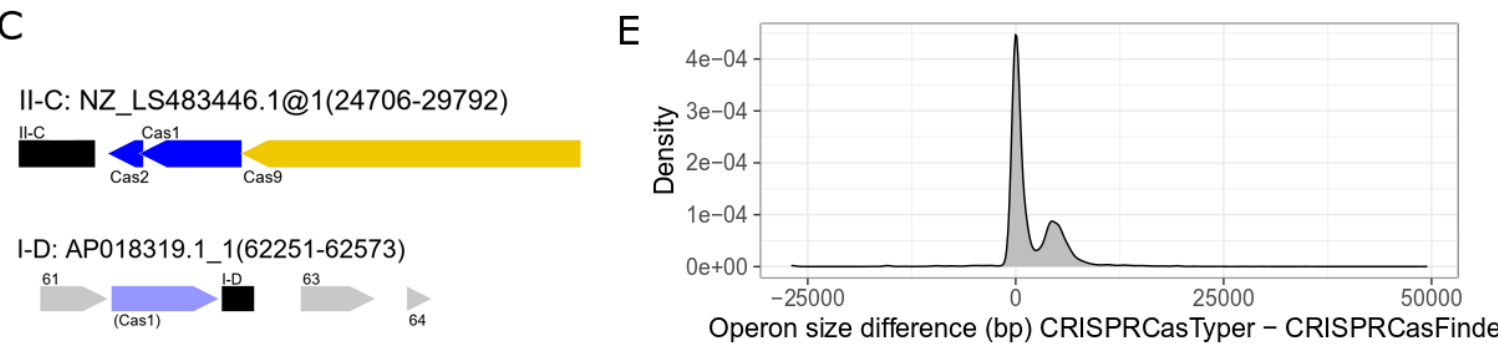

Hybrid(I-F,III-B): NZ_CP022741.1@31(1572959-1591163)
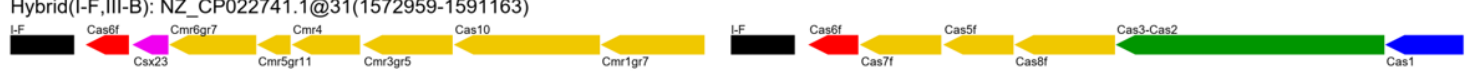

Figure 1. A) CRISPRCasTyper workflow. B) Prediction matrix of CRISPR repeat typer against an unseen test dataset. Only subtypes with at least 20 repeats were included in the model. Counts are normalized per row totals. C) Examples of graphical outputs from CRISPRCasTyper. The predicted subtype associated with a repeat sequence is written above the array, colored in black (top). Interference module in yellow (except Cas6), adaptation module in blue, Cas6 in red, and accessory genes in purple. Unknown genes (grey) and genes with low-quality matches (same color scheme in lighter shade) can be added to the plots, also around orphan CRISPR arrays (middle). Furthermore, CRISPRCasTyper resolves hybrid systems (bottom). D) Performance of CRISPRCasTyper 
versus CRISPRCasFinder on manually curated CRISPR-Cas systems. 'Correct with CRISPR' denotes loci which were resolved using subtype prediction based on the CRISPR repeat sequence. Asterisks $\left(^{*}\right)$ denote subtypes not included in CRISPRCasFinder; CRISPRCasFinder includes type IV without subtype prediction, and these are denoted as 'Ambiguous'. E) Density plot of differences in operon sizes between CRISPRCasTyper and CRISPRCasFinder.

\section{Benchmark}

CRISPRCasTyper was compared with the widely used CRISPRCasFinder (Couvin et al. 2018) using the manually curated CRISPR-Cas loci from Makarova et al. 2020 (Makarova et al. 2020), the newest type IV classification from Pinilla-Redondo et al. 2019 (Pinilla-Redondo et al. 2019) and manually curated III-E loci (see Methods and Materials). We find that CRISPRCasTyper outperforms CRISPRCasFinder at identifying 17 subtypes and is equally accurate in the prediction of the remaining 2 subtypes included in CRISPRCasFinder (Fig. 1D) (Couvin et al. 2018). Across these 19 subtypes, CRISPRCasTyper had a median accuracy of $99.5 \%$, while that of CRISPRCasFinder was $93.9 \%$. The median accuracy of CRISPRCasTyper on all 31 subtypes (12 lacking in CRISPRCasFinder) was $98.6 \%$. Furthermore, CRISPRCasTyper often provides a more complete cas operon identification (Fig. 1E). An example is a type III-B operon in an Acidilobus saccharovorans genome (NC_014374.1), in which CRISPRCasFinder identifies cmr4, cmr5, and cmr6, whereas CRISPRCasTyper finds 9 additional genes, including cas1, cas2, cas4, cas 10, and cas 6 .

Both CRISPRCasTyper and CRISPRCasFinder found few false positives (28 (0.4\%) and 24 $(0.4 \%)$, respectively), with a large bias towards VI-B (10 and 13 , respectively). Interestingly, several of these seem to be true positives, which have been missed in the curated dataset; 9 of the VI-B operons have adjacent CRISPR arrays whose repeat sequence is predicted by CRISPRCasTyper to be VI-B associated (Table S1). V-F is especially challenging due to the similarity of its effector to transposases; we chose a conservative approach, which identifies as many as possible without finding false positives. Many $\mathrm{V}-\mathrm{F}$ are missed with this approach, but some can be found using the graphical output if they are adjacent to CRISPR arrays. When the V-F subtype is more clearly defined, improved HMMs might solve this problem.

\section{Methods and Materials}

\section{CRISPRCasTyper}

Open reading frames are called with prodigal v2.6.3 (Hyatt et al. 2010). Protein-profile alignments are performed with HMMER3 (Eddy 2009). HMM matches are filtered in a two-step process. All single-effector genes (cas9, cas12, cas13, gRAMP) are filtered with specific cutoffs (see Table S2). The remaining Cas proteins are filtered with an E-value cutoff of 0.01 , and sequence and HMM coverage both of $30 \%$. The single-effector cutoffs were set by running a grid search across the curated set with coverages between $5 \%$ and $95 \%$ with a step size of $5 \%$, and E-values from $10 \mathrm{e}-5$ to $1 \mathrm{e}-150$ with a step size of the exponent of 5 . The subtypes with no representative in the curated set were given the following cutoffs: E-value: 1e-5, sequence coverage: $90 \%$, HMM coverage: $25 \%$. Cas genes 
are grouped into operons based on synteny. By default, no more than 3 unannotated genes can separate known cas genes to be considered part of a single operon.

The operons are then typed based on a scoring scheme where each Cas HMM within it has a score between 0 and 4 for each subtype. The scoring scheme was built such that mandatory HMMs give a score of 2 and accessory genes a score of 1 . HMMs specific to a subtype adds 2 to the score. Forbidden HMMs give a score of -4 . The subtype that obtains the highest score is then assigned to the operon if the score is at least 6 and there are at least 3 different cas genes. Operons with at least 6 cas genes and two or more types with a score of at least 6 and at least one specific HMM are denoted as 'Hybrid' systems. Operons falling outside these cutoffs are annotated as 'Putative' unless one of the genes is a Class 2 effector or the III-E gRAMP fusion protein. Non-hybrid operons with multiple equally scoring subtypes are annotated as 'Ambiguous'.

To resolve problems of ambiguity, a score of 0.1 was added to mandatory and specific I-F HMMs, such that typing defaults to type I-F, unless TniQ is found which will change the type to I-F_T, transposon-associated I-F. Similarly for IV-A2, which is distinguished from IV-A1 and IV-A3 by the absence of Csf1. Typing therefore defaults to IV-A2 unless a Csf1 is found or Csf2 or Csf4 is specific to IV-A1 or IV-A3. Similarly with III-A and III-F, for which the scores are designed to default to III-A unless SSgr11 is detected. As Cas12j and Cas12k are so similar the typing defaults to $\mathrm{V}-\mathrm{J}$ unless transposon-associated TniQ, TnsB, and TnsC are found.

\section{RepeatTyper}

A curated set of subtyped repeat sequences was created by predicting CRISPR arrays with minced v0.4.2 (https://github.com/ctSkennerton/minced, Bland et al. 2007) in the curated datasets (Makarova et. al. 2020; Pinilla-Redondo et. al. 2019). Consensus repeats from all arrays within $1 \mathrm{kbp}$ to a cas operon were included. This resulted in a total of 5838 subtyped repeat sequences. Only subtypes with at least 20 repeat sequences were included in the model. For each repeat sequence all canonical tetramers were counted, and these 136 features were the input for our model. The sequences were split in $70 \%$ training data, used to train the model and choose parameters, and $30 \%$ testing data, used as an unseen dataset to evaluate the accuracy of the final model. We used xgboost v1.0.2 with multi:softprob objective evaluated with mlogloss and 3 -fold cross-validation across a grid of max-depth $=\{4$, $6,8\}$, subsample $=\{0.6,0.8,1\}$, and colsample_bytree $=\{0.6,0.8,1\}$. The models were run with a learning rate of 0.3 for 100 boosting rounds, but with 10 early stopping rounds. The remaining parameters were defaults. The script for training this model is part of CRISPRCasTyper, such that users can easily re-run the model on their own repeat-set and/or with other parameters. The accuracy for each subtype was calculated as percent correct predictions on the test set. The adjusted accuracy was then the average accuracy across all subtypes, such that subtypes with many repeats were not inflating the accuracy.

On the web server, consensus repeats from novel loci are automatically included in the model. Novelty is based on position of loci in the sequence, the subtype prediction, and the repeat sequence. This updated set was supplemented with subtyped repeats from Ensembl bacterial and archaeal genomes (Yates et al. 2020), GTDB (Parks et al. 2018), and NCBI metagenomes (ftp://ftp.ncbi.n/m.nih.gov/genomes/genbank/metagenomes/). As of writing this article, the model includes 40717 subtyped repeats across 37 subtypes/variants; 26 of 
these with at least 20 repeat sequences. This updated model is automatically re-trained each month with the same parameters as above except with $\max$-depth $=\{6,8,10\}$.

\section{Hidden Markov Models}

CRISPRCasTyper includes 680 HMMs. The largest share was built using hmmbuild from HMMER3 v3.2.1 (Eddy 2009) on the multiple alignments provided by Makarova et al. 2020 (Makarova et al. 2020), excluding the consensus sequence. Some HMMs were obtained from CRISPRCasFinder 2.0.2. The V-K specific HMMs and the I-F associated TniQ HMM were built from multiple alignments created with MUSCLE v3.8.1551 (Edgar 2004), based on previous studies (Strecker et al. 2019; Klompe et al. 2019). Type IV HMMs were obtained from Pinillia-Redondo et. al. 2019.

\section{Benchmark}

All non-type IV CRISPR-Cas loci from Makarova et al. 2020, available at ftp://ftp.ncbi.nih.gov/pub/wolf/ suppl/CRISPRclass19/, were included. Multi-systems and partial systems were excluded from the benchmark. Type IV loci were obtained from Pinilla-Redondo et. al. 2019 to include the newest classification. We further included a curated set of III-E loci (Table S3). For CRISPRCasTyper, all predicted non-putative systems were included. For CRISPRCasFinder, all systems, including partial systems, were included. As Makarova et al. 2020 does not include V-K, the few V-J operons which were predicted by CRISPRCasTyper to be $\mathrm{V}-\mathrm{K}$ were labelled as correct classifications. For determining false positives only operons not overlapping with any loci, including partial and multi-systems, were counted. CRISPRCasFinder version 4.2.17 with CasFinder 2.0.2 was used for the benchmark. CRISPRCasTyper version 1.0.0 was used for the benchmark.

\section{Data availability}

The scoring table, all HMM profiles, the filtering cut-offs, the repeat typer model, and the completion files are available in the data directory at the CRISPRCasTyper github: https://github.com/Russel88/CRISPRCasTyper/tree/master/data. The updated repeat typer models are available at http://mibi.galaxy.bio.ku.dk/russel/repeattyper/.

\section{Web server}

The web server was built on the jobson framework (https://github.com/adamkewley/jobson), with a modified UI available at https://github.com/Russel88/jobson. The web server also includes the possibility to submit a RefSeq/Genbank accession, which will download the corresponding nucleotide sequence through the entrez-direct command line tool.

\section{Acknowledgments}

We would like to thank Sarah Camara for testing the software and providing valuable feedback. J.R and S.A.S were supported by the Novo Nordisk Foundation. R.P-R was financed by the Independent Research Fund Denmark, InTrans Project [\#8022-00322B]. D.M-M was supported by a University of Otago Doctoral Scholarship. Part of the data analysis was performed on Computerome, the Danish National Computer for Life Sciences. 


\section{References}

Barrangou, Rodolphe, and Jennifer A. Doudna. 2016. "Applications of CRISPR Technologies in Research and beyond." Nature Biotechnology.

Bernheim, Aude, David Bikard, Marie Touchon, and Eduardo P. C. Rocha. 2020. "Atypical Organizations and Epistatic Interactions of CRISPRs and Cas Clusters in Genomes and Their Mobile Genetic Elements." Nucleic Acids Research 48 (2): 748-60.

Chen, Tianqi, and Carlos Guestrin. 2016. "XGBoost: A Scalable Tree Boosting System." In Proceedings of the 22nd ACM SIGKDD International Conference on Knowledge Discovery and Data Mining, 11:785-94. New York, NY, USA: ACM.

Couvin, David, Aude Bernheim, Claire Toffano-Nioche, Marie Touchon, Juraj Michalik, Bertrand Néron, Eduardo P. C. Rocha, Gilles Vergnaud, Daniel Gautheret, and Christine Pourcel. 2018. "CRISPRCasFinder, an Update of CRISRFinder, Includes a Portable Version, Enhanced Performance and Integrates Search for Cas Proteins." Nucleic Acids Research 46 (W1): W246-51.

Crawley, Alexandra B., James R. Henriksen, and Rodolphe Barrangou. 2018. "CRISPRdisco: An Automated Pipeline for the Discovery and Analysis of CRISPR-Cas Systems." The CRISPR Journal 1 (April): 171-81.

Eddy, Sean R. 2009. "A New Generation of Homology Search Tools Based on Probabilistic Inference." Genome Informatics. International Conference on Genome Informatics 23 (1): 205-11.

Edgar, R. C. 2004. "MUSCLE: Multiple Sequence Alignment with High Accuracy and High Throughput." Nucleic Acids Research.

Hampton, Hannah G., Bridget N. J. Watson, and Peter C. Fineran. 2020. "The Arms Race between Bacteria and Their Phage Foes." Nature 577 (7790): 327-36.

Hille, Frank, Hagen Richter, Shi Pey Wong, Majda Bratovič, Sarah Ressel, and Emmanuelle Charpentier. 2018. "The Biology of CRISPR-Cas: Backward and Forward." Cell 172 (6): 1239-59.

Hyatt, Doug, Gwo-Liang Chen, Philip F. Locascio, Miriam L. Land, Frank W. Larimer, and Loren J. Hauser. 2010. "Prodigal: Prokaryotic Gene Recognition and Translation Initiation Site Identification." BMC Bioinformatics 11 (March): 119

Jackson, Simon A., Rebecca E. McKenzie, Robert D. Fagerlund, Sebastian N. Kieper, Peter C. Fineran, and Stan J. J. Brouns. 2017. "CRISPR-Cas: Adapting to Change." Science 356 (6333).

Klompe, Sanne E., Phuc L. H. Vo, Tyler S. Halpin-Healy, and Samuel H. Sternberg. 2019. "Transposon-Encoded CRISPR-Cas Systems Direct RNA-Guided DNA Integration." Nature.

Koonin, Eugene V., and Kira S. Makarova. 2019. "Origins and Evolution of CRISPR-Cas Systems." Philosophical Transactions of the Royal Society of London. Series B, Biological Sciences 374 (1772): 20180087.

Lange, Sita J., Omer S. Alkhnbashi, Dominic Rose, Sebastian Will, and Rolf Backofen. 2013. "CRISPRmap: An Automated Classification of Repeat Conservation in Prokaryotic Adaptive Immune Systems." Nucleic Acids Research 41 (17): 8034-44.

Makarova, Kira S., Yuri I. Wolf, Omer S. Alkhnbashi, Fabrizio Costa, Shiraz A. Shah, Sita J. Saunders, Rodolphe Barrangou, et al. 2015. "An Updated Evolutionary Classification of CRISPR-Cas Systems." Nature Reviews. Microbiology 13 (September): 722.

Makarova, Kira S., Yuri I. Wolf, Jaime Iranzo, Sergey A. Shmakov, Omer S. Alkhnbashi, Stan J. J. Brouns, Emmanuelle Charpentier, et al. 2020. "Evolutionary Classification of CRISPR-Cas Systems: A Burst of Class 2 and Derived Variants." Nature Reviews. Microbiology 18 (2): 67-83.

Makarova, Kira S., Yuri I. Wolf, and Eugene V. Koonin. 2018. "Classification and Nomenclature of CRISPR-Cas Systems: Where from Here?" The CRISPR Journal 1 (5): 325-36.

Mohanraju, Prarthana, Kira S. Makarova, Bernd Zetsche, Feng Zhang, Eugene V. Koonin, and John van der Oost. 2016. "Diverse Evolutionary Roots and Mechanistic Variations of the CRISPR-Cas Systems." Science 353 (6299): aad5147.

Pickar-Oliver, Adrian, and Charles A. Gersbach. 2019. "The next Generation of CRISPR-Cas Technologies and Applications." Nature Reviews Molecular Cell Biology.

Pinilla-Redondo, Rafael, David Mayo-Muñoz, Jakob Russel, Roger A. Garrett, Lennart Randau, Søren J. Sørensen, and Shiraz A. Shah. 2019. "Type IV CRISPR-Cas Systems Are Highly Diverse and Involved in Competition between Plasmids." Nucleic Acids Research.

Strecker, Jonathan, Alim Ladha, Zachary Gardner, Jonathan L. Schmid-Burgk, Kira S. Makarova, Eugene V. Koonin, and Feng Zhang. 2019. "RNA-Guided DNA Insertion with CRISPR-Associated Transposases." Science.

Yates, Andrew D., Premanand Achuthan, Wasiu Akanni, James Allen, Jamie Allen, Jorge Alvarez-Jarreta, M. Ridwan Amode, et al. 2020. "Ensembl 2020." Nucleic Acids Research 48 (D1): D682-88. 


\section{Supplementary Information}

\section{Prediction}

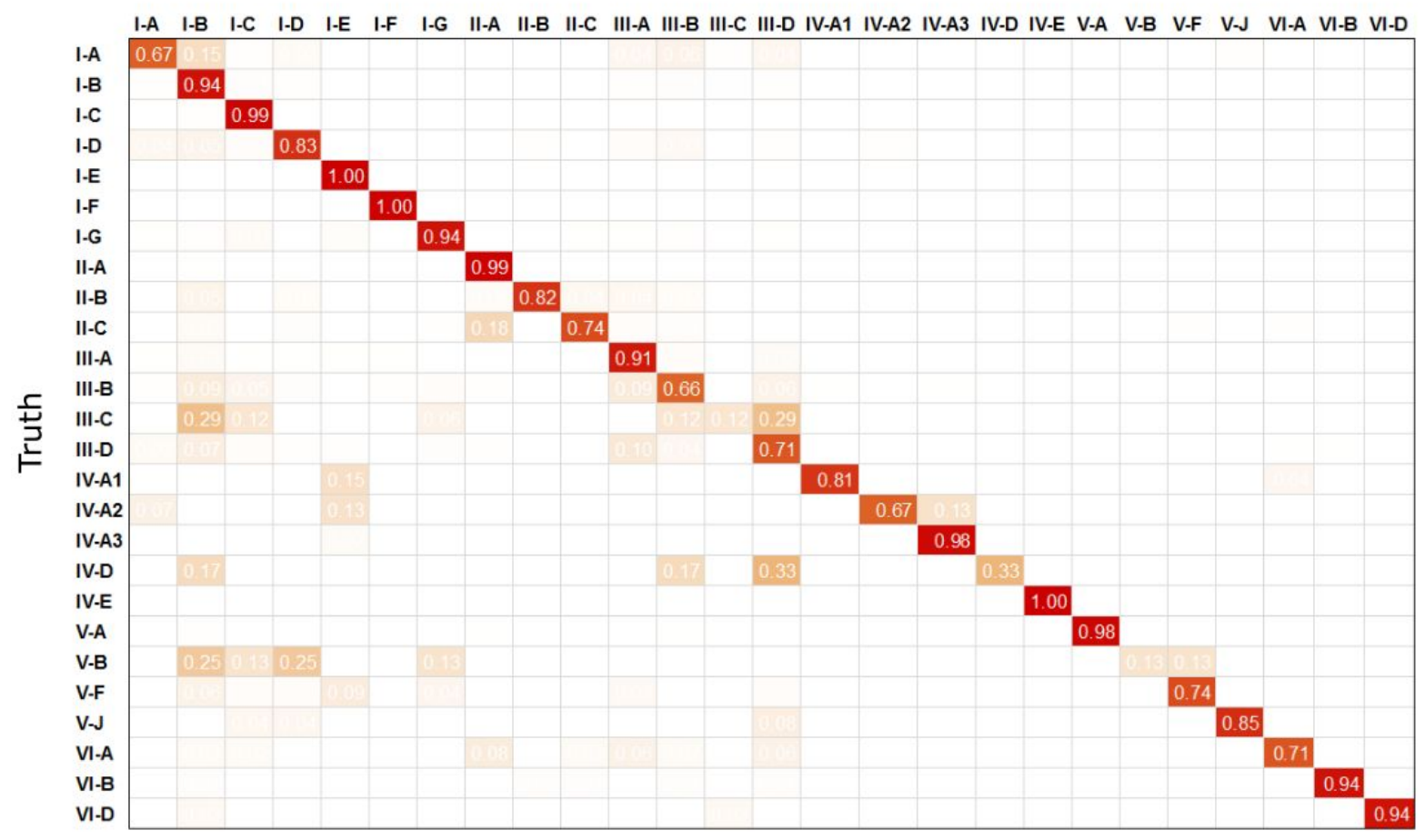

Figure S1. Prediction matrix of CRISPR repeat typer against an unseen test dataset using the updated repeat set of $40 \mathrm{k}$ repeat sequences. Counts are normalized per row totals, such that the diagonals contain the accuracy for each subtype. 
Table S1. False positive operons from CRISPRCasTyper. Several have CRISPRs adjacent, and the predicted subtype of these CRISPRs match the CRISPRCasTyper predictions. * The subtype for the repeat sequence from the V-J on NZ_AP014815.1 could not be predicted with the curated repeat set, but was predicted to be $\mathrm{V}-\mathrm{J}$ with the updated repeat set. $\S$ This I-E loci is likely part of the I-E loci in the end of this sequence.

\begin{tabular}{|c|c|c|c|c|c|}
\hline Nucleotide & Subtype & Start & End & CRISPR & CRISPR Subtype \\
\hline NC_014377.1 & I-A & 1179284 & 1183097 & No & \\
\hline NC_017768.1 & I-A & 838904 & 841348 & No & \\
\hline NZ_CP022385.1 & I-A & 1996653 & 2002119 & No & \\
\hline NZ_CP031218.1 & I-B & 1882001 & 1884693 & No & \\
\hline NZ_CP019794.1 & I-C & 4407864 & 4411147 & Yes & I-C \\
\hline NC_008750.1 & I-D & 2011041 & 2015216 & No & \\
\hline NC_009438.1 & I-D & 2601641 & 2605816 & No & \\
\hline NC_014248.1 & I-D & 4303670 & 4309589 & No & \\
\hline NC_017566.1 & I-D & 2602859 & 2607034 & No & \\
\hline NC_015499.1 & I-E & 1031980 & 1036308 & No & \\
\hline NZ_CP032329.1 & I-E § & 3 & 7027 & Yes & I-E \\
\hline NZ_CP032099.1 & II-B & 801907 & 802326 & No & \\
\hline NC_015519.1 & III-A & 3080 & 4920 & No & \\
\hline NC_019954.2 & III-A & 3199 & 5039 & No & \\
\hline NZ_CP021838.1 & III-B & 2057 & 4497 & No & \\
\hline NC_002950.2 & VI-B & 1244197 & 1248317 & Yes & VI-B \\
\hline NC_010729.1 & VI-B & 1410847 & 1414967 & Yes & VI-B \\
\hline NC_014734.1 & VI-B & 3133284 & 3136748 & No & \\
\hline NC_016001.1 & VI-B & 2545026 & 2549183 & Yes & VI-B \\
\hline NZ_CP011995.1 & VI-B & 920122 & 924242 & Yes & VI-B \\
\hline NZ_CP012889.1 & VI-B & 1409775 & 1413895 & Yes & VI-B \\
\hline NZ_CP024591.1 & VI-B & 209480 & 213600 & Yes & VI-B \\
\hline NZ_CP024595.1 & VI-B & 1078280 & 1082400 & Yes & VI-B \\
\hline NZ_CP025930.1 & VI-B & 1411695 & 1415815 & Yes & VI-B \\
\hline NZ_CP025932.1 & VI-B & 1167344 & 1171464 & Yes & VI-B \\
\hline NC_019678.1 & V-J & 6308425 & 6308943 & No & \\
\hline NZ_AP014815.1 & V-J & 2551679 & 2553529 & Yes & Unknown (V-J)* \\
\hline NZ_CP026681.1 & V-J & 5056609 & 5057136 & No & \\
\hline
\end{tabular}


Table S2. Specific cutoffs used for filtering single-effector cas genes.

\begin{tabular}{|l|r|r|r|}
\hline Gene & E-value & Coverage Sequence & \multicolumn{1}{l|}{ Coverage HMM } \\
\hline cas9 & $1.00 \mathrm{E}-10$ & 0.35 & 0.1 \\
\hline cas12a & $1.00 \mathrm{E}-05$ & 0.9 & 0.75 \\
\hline cas12b & $1.00 \mathrm{E}-10$ & 0.9 & 0.35 \\
\hline cas12c & $1.00 \mathrm{E}-05$ & 0.9 & 0.25 \\
\hline cas12d & $1.00 \mathrm{E}-05$ & 0.9 & 0.25 \\
\hline cas12e & $1.00 \mathrm{E}-05$ & 0.9 & 0.25 \\
\hline cas12f & $1.00 \mathrm{E}-130$ & 0.8 & 0.7 \\
\hline cas12g & $1.00 \mathrm{E}-05$ & 0.9 & 0.25 \\
\hline cas12h & $1.00 \mathrm{E}-05$ & 0.9 & 0.25 \\
\hline cas12i & $1.00 \mathrm{E}-05$ & 0.9 & 0.25 \\
\hline cas12j & $1.00 \mathrm{E}-05$ & 0.9 & 0.25 \\
\hline cas12k & $1.00 \mathrm{E}-05$ & 0.9 & 0.25 \\
\hline cas13a & $1.00 \mathrm{E}-10$ & 0.05 & 0.55 \\
\hline cas13b & $1.00 \mathrm{E}-10$ & 0.95 & 0.85 \\
\hline cas13c & $1.00 \mathrm{E}-10$ & 0.9 & 0.75 \\
\hline cas13d & $1.00 \mathrm{E}-10$ & 0.95 & 0.95 \\
\hline gRAMP & $1.00 \mathrm{E}-10$ & 0.75 & 0.75 \\
\hline
\end{tabular}

Table S3. Accession numbers of III-E gRAMP proteins. No adjacent CRISPR could be determined (NA) in short contigs or with a gRAMP gene at the end of a sequence.

\begin{tabular}{|l|l|c|}
\hline Nucleotide & Gene & CRISPR \\
\hline JRYO01000185.1 & KHE91659.1 & Yes \\
\hline NZ_BAFH01000003.1 & WP_007220849.1 & Yes \\
\hline MVRP01000104.1 & OPY65763.1 & Yes \\
\hline MGTA01000040.1 & OGR07205.1 & Yes \\
\hline NZ_BEXT01000001.1 & WP_124327589.1 & Yes \\
\hline LAQJ01000233.1 & KKO18793.1 & NA \\
\hline NBMK01000156.1 & OQY58162.1 & NA \\
\hline QMMU01000439.1 & RLC14096.1 & NA \\
\hline QMMU01000323.1 & RLC15988.1 & NA \\
\hline QMMU01000137.1 & RLC19861.1 & NA \\
\hline JPDT01001326.1 & KPA14974.1 & No \\
\hline NZ_BEXT01000001.1 & WP_124327589.1 & No \\
\hline
\end{tabular}

